\title{
PLANE POLOIDAL-TOROIDAL DECOMPOSITION OF DOUBLY PERIODIC VECTOR FIELDS. PART 2. THE STOKES EQUATIONS
}

\author{
G. D. MCBAIN ${ }^{1}$
}

(Received 31 March, 2004; revised 3 May, 2005)

\begin{abstract}
We continue our study of the adaptation from spherical to doubly periodic slot domains of the poloidal-toroidal representation of vector fields. Building on the successful construction of an orthogonal quinquepartite decomposition of doubly periodic vector fields of arbitrary divergence with integral representations for the projections of known vector fields and equivalent scalar representations for unknown vector fields (Part 1), we now present a decomposition of vector field equations into an equivalent set of scalar field equations. The Stokes equations for slow viscous incompressible fluid flow in an arbitrary force field are treated as an example, and for them the application of the decomposition uncouples the conservation of momentum equation from the conservation of mass constraint. The resulting scalar equations are then solved by elementary methods. The extension to generalised Stokes equations resulting from the application of various time discretisation schemes to the Navier-Stokes equations is also solved.
\end{abstract}

\section{Introduction}

In Part 1 [8] an orthogonal decomposition of doubly periodic (d.p.) vector fields into five parts, three of which are divergence-free, was derived. Associated with this quinquepartite decomposition was a representation of such fields in terms of scalars. An advantage of this representation is that unknown vector fields known to be divergencefree can automatically be represented as such simply by setting to zero the scalars associated with the two non-divergence-free subspaces.

In Part 2 (Section 2) the quinquepartite decomposition is used to derive a set of scalar equations equivalent to a d.p. vector field equation $f=0$. The derivation uses the equivalence between the vanishing of $f$ and the vanishing of its projections in each of the five subspaces.

\footnotetext{
'School of Aerospace, Mechanical and Mechatronic Engineering, The University of Sydney, Australia: e-mail: geordie.mcbain@aeromech.usyd.edu.au.

(C) Australian Mathematical Society 2005, Serial-fee code 1446-1811/05
} 
As an example, in Section 3 we treat the Stokes equations for viscous fluid flow between two solid parallel walls under an arbitrary body force. The equivalent scalar equations can then be solved by elementary methods.

An appendix treats a generalised Stokes problem.

\subsection{Stokes equations The Navier-Stokes equations}

$$
\begin{aligned}
\operatorname{Re}\left(\frac{\partial u}{\partial t}+u \cdot \nabla u\right) & =-\nabla P+b+\nabla^{2} u, \\
\nabla \cdot u & =0
\end{aligned}
$$

describe the evolution in time $t$ of the flow of incompressible fluid with velocity $u$ and pressure $P$ subject to a body force $b$. The nonnegative Reynolds number $\operatorname{Re}$ signifies the relative strength of inertial and viscous forces. Setting $\operatorname{Re}=0$ gives the Stokes equations. The Stokes equations are of interest because 'as is well known, the study of the properties of solutions to the Stokes system plays an essential role in the mathematical theory of viscous fluid flows governed by the Navier-Stokes equations' [5].

Also, the Stokes equations asymptotically describe very slow flows of very viscous fluids on very short length scales. Further, Stokes equations with known $\boldsymbol{b} \neq \mathbf{0}$ arise in asymptotic expansions for the Navier-Stokes equations at small $\mathrm{Re}$, not merely for the zeroth order $\mathscr{O}(1)$ term, but, in bounded domains, for all orders [10-12].

Equations closely related to the Stokes equations like

$$
\begin{aligned}
\alpha u & =-\nabla P+b+\nabla^{2} u, \\
\nabla \cdot u & =0
\end{aligned}
$$

even arise in numerical methods for solving Navier-Stokes problems at arbitrary Reynolds numbers. When taking into account implicitly the viscous term and explicitly the advection term, the time discretisation results in a generalised Stokes problem (1.1)-(1.2) to be solved at each time-step [14,15]. The same equations result from various operator splitting techniques $[3,19]$. Thus, this extension to the Stokes problem is relevant to our longer term aim of developing a Navier-Stokes solver based on the quinquepartite decomposition.

\section{Scalar equivalents of vector field equations}

In Section 2, a set of scalar equations equivalent to the d.p. vector field equation $f(x, y, z)=\mathbf{0}$ is derived. 
2.1. Norms Since the five projections are mutually orthogonal and collectively complete, we have the Pythagorean equation

$$
\|f\|^{2}=\left\|\mathrm{P}_{\mathscr{N}} f\right\|^{2}+\left\|\mathrm{P}_{\mathscr{D}} f\right\|^{2}+\left\|\mathrm{P}_{\mathscr{S}} f\right\|^{2}+\left\|\mathrm{P}_{\mathscr{P}} f\right\|^{2}+\left\|\mathrm{P}_{\mathscr{T}} f\right\|^{2} .
$$

The squares of the norms here are (in terms of the scaloidal, poloidal and toroidal potentials of $f: \sigma, \Psi$ and $\tau$ )

$$
\begin{aligned}
\left\|\mathrm{P}_{\mathscr{N}} f\right\|^{2} & =\left\|\left\langle f_{x}\right\rangle_{\square}-\left\langle f_{x}\right\rangle\right\|^{2}, \\
\left\|\mathrm{P}_{\mathscr{B}} f\right\|^{2} & =\left\|\left\langle f_{x}\right\rangle\right\|^{2}+\left\|\left\langle f_{y}\right\rangle_{\square}\right\|^{2}+\left\|\left\langle f_{z}\right\rangle_{\square}\right\|^{2}, \\
\left\|\mathrm{P}_{\mathscr{S}} f\right\|^{2} & =\|\nabla \sigma\|^{2}, \\
\left\|\mathrm{P}_{\mathscr{P}} f\right\|^{2} & =\left\|\nabla_{\square}^{2} \Psi\right\|^{2}+\left\|\nabla_{\square} \mathrm{D} \Psi\right\|^{2} \quad \text { and } \\
\left\|\mathrm{P}_{\mathscr{T}} f\right\|^{2} & =\|\boldsymbol{\Lambda} \tau\|^{2} .
\end{aligned}
$$

The scaloidal (2.4), poloidal (2.5) and toroidal (2.6) square-norms can be expressed alternatively as

$$
\begin{aligned}
\left\|\mathrm{P}_{\mathscr{S}} f\right\|^{2} & =\left\langle\sigma, S_{\mathscr{S}} f\right\rangle \\
\left\|\mathrm{P}_{\mathscr{P}} f\right\|^{2} & =\left\langle\Psi, S_{\mathscr{P}} f\right\rangle-\left(x_{1}-x_{0}\right)^{-1}\left[\left\langle\Psi, \nabla_{\square} \cdot f\right\rangle_{\square}\right]_{x_{0}}^{x_{1}} \text { and } \\
\left\|\mathrm{P}_{\mathscr{T}} f\right\|^{2} & =\left\langle\tau, S_{\mathscr{T}} f\right\rangle
\end{aligned}
$$

by defining the scalar operators

$$
\begin{aligned}
& \mathrm{S}_{\mathscr{S}} f=\nabla \cdot f-\langle\nabla \cdot f\rangle_{\square}, \\
& \mathrm{S}_{\mathscr{P}} f=-\nabla_{\square}^{2} f_{x}+D \nabla_{\square} \cdot f \quad \text { and } \\
& \mathrm{S}_{\mathscr{T}} f=\Lambda \cdot f .
\end{aligned}
$$

In Section 2.2 we use the Pythagorean equation (2.1) to reduce a vector field equation to an equivalent set of scalar equations.

2.2. Decomposition of vector field equations It follows from the Pythagorean equation (2.1) that the vector field equation

$$
\boldsymbol{f}(x, y, z)=f\left(x, y+\frac{2 \pi}{k \cos \gamma}, z+\frac{2 \pi}{k \sin \gamma}\right)=\mathbf{0}
$$

is equivalent to $\|\mathrm{P} f\|=0$ for $\mathrm{P}=\mathrm{P}_{\mathscr{N}}, \mathrm{P}_{\mathscr{B}}, \mathrm{P}_{\mathscr{S}}, \mathrm{P}_{\mathscr{P}}$ and $\mathrm{P}_{\mathscr{T}}$; however, it is not necessary to actually carry out the projections, as shown by the following theorem. 
THEOREM 2.1. The d.p. vector field equation $f=0$ is equivalent to the seven d.p. scalar field equations

$$
\begin{aligned}
\left(f_{x}\right\rangle_{\square}-\left\langle f_{x}\right\rangle & =0, \\
\left\langle f_{x}\right\rangle=\left\langle f_{y}\right\rangle_{\square}=\left\langle f_{z}\right\rangle_{\square} & =0, \\
S_{\mathscr{S}} f & =0, \\
S_{\mathscr{P}} f & =0 \quad \text { and } \\
S_{\mathscr{T}} f & =0
\end{aligned}
$$

and the end conditions

$$
-\nabla_{\square} \cdot f=0 \quad\left(x=x_{0}, x_{1}\right)
$$

PRoof. From (2.2), the vanishing of the $\mathscr{N}$-norm is equivalent to (2.11).

From (2.3), a zero $\mathscr{B}$-part is equivalent to (2.12).

The sufficiency of (2.13)-(2.16) is evident from the alternate expressions for the norms (2.7)-(2.9).

Their necessity follows from the uniqueness of the potentials $\sigma, \Psi$ and $\tau$ (Part 1); for example, $\mathrm{P}_{\mathscr{S}} f=0$ implies $\sigma=0$ so that (2.13) follows from the definition of $\mathrm{S}_{\mathscr{S}} f(2.10)$, with similar results for the poloidal and toroidal parts. The vanishing of $\Psi$ throughout $x_{0}<x<x_{1}$ also implies (2.16), by the boundary condition on the Poisson equation for the poloidal potential.

2.3. Fourier decomposition of vector field equations The scaloidal, poloidal and toroidal subspaces can be further partitioned with the trigonometric Fourier decomposition. The associated norms are

$$
\begin{aligned}
& \left\|\mathrm{P}_{\mathscr{S}_{\ell} v}\right\|^{2}=\left\langle\tilde{\sigma}_{\ell}, S_{\mathscr{S}_{\ell} v} v\right\rangle \text {, } \\
& \left\|\mathrm{P}_{\mathscr{S}_{\mathfrak{l}}, v}\right\|^{2}=\left\langle\widetilde{\Psi}_{\ell}, S_{\mathscr{S}_{l}} v\right\rangle+\left(x_{1}-x_{0}\right)^{-1}\left[\left\langle\widetilde{\Psi}_{\ell}, \kappa_{\ell}^{2} \mathrm{D} \widetilde{\Psi}_{\ell}\right\rangle_{\square}\right]_{x_{0}}^{x_{1}} \quad \text { and } \\
& \left\|P_{\mathscr{T}}, v\right\|^{2}=\left\langle\tilde{\tau}_{\ell}, S_{\mathscr{T}_{\ell}} v\right\rangle \text {, }
\end{aligned}
$$

where

$$
\begin{aligned}
S_{\mathscr{S}_{l} v} & \equiv\left\langle e_{\ell}, S_{\mathscr{S}} v\right\rangle_{\square}=\left(\kappa_{\ell}^{2}-D^{2}\right) \tilde{\sigma}_{\ell}, \\
S_{\mathscr{P}_{\ell}} v & \equiv\left\langle e_{\ell}, S_{\mathscr{S}} v\right\rangle_{\square}=\kappa_{l}^{2}\left(\kappa_{\ell}^{2}-D^{2}\right) \tilde{\Psi} \quad \text { and } \\
S_{\mathscr{T}_{l}} v & \equiv\left\langle e_{\ell}, S_{\mathscr{T}} v\right\rangle_{\square}=\kappa_{\ell}^{2} \tilde{\tau}_{\ell} .
\end{aligned}
$$

Then in (2.1) the last three terms can be replaced with

$$
\sum_{l}^{\prime}\left(\left\|\mathrm{P}_{\mathscr{S}_{l}} f\right\|^{2}+\left\|\mathrm{P}_{\mathscr{P}_{l}} f\right\|^{2}+\left\|\mathrm{P}_{\mathscr{T}_{l}} f\right\|^{2}\right)
$$


(a prime being inserted above the summation when the term for which $l_{y}=l_{z}=0$ is to be omitted) and the last four equations of Theorem 2.1 by

$$
\begin{aligned}
& \mathrm{S}_{\mathscr{S}_{\ell}} f=\mathrm{D}\left\langle e_{\ell}, f_{x}\right\rangle_{\square}+\mathrm{i} \kappa_{\ell}\left\langle e_{\ell}, f_{\eta_{\ell}}\right\rangle_{\square}=0, \\
& \mathrm{~S}_{\mathscr{P}_{\ell}} f=\kappa_{\ell}^{2}\left\langle e_{\ell}, f_{x}\right\rangle_{\square}+\mathrm{i} \kappa_{\ell} \mathrm{D}\left\langle e_{\ell}, f_{\eta_{\ell}}\right\rangle_{\square}=0 \quad \text { and } \\
& \mathrm{S}_{\mathscr{T}_{\ell}} f=\mathrm{i} \kappa_{\ell}\left\langle e_{\ell}, f_{\zeta_{\ell}}\right\rangle_{\square}=0
\end{aligned}
$$

for all $\ell \neq 0$. At the ends $\left(x=x_{0}, x_{1}\right)$

$$
\left\langle e_{\ell},-\nabla_{\square} \cdot f\right\rangle_{\square}=-\mathrm{i} \kappa_{\ell}\left\langle e_{\ell}, f_{\eta_{\ell}}\right\rangle_{\square}=0 .
$$

\section{The Stokes equations}

The Stokes equations for creeping flow of an incompressible fluid with a body force $b$ are

$$
\begin{aligned}
0 & =-\nabla P+b+\nabla^{2} u, \\
\nabla \cdot u & =0
\end{aligned}
$$

subject to appropriate conditions at the ends; for example, prescribed $u$. In this section we derive the solution of (3.1)-(3.2); the solution of the generalised Stokes problem (1.1)-(1.2) is given in Appendix A.

3.1. Representation of velocity and pressure The incompressibility constraint (3.2) implies the existence of the Schmitt-Wahl mean-poloidal-toroidal representation [8, 17]

$$
\boldsymbol{u}=U \mathbf{i}_{x}+V \mathbf{i}_{y}+W \mathbf{i}_{z}-\nabla_{\square}^{2} \Psi \mathbf{i}_{x}+\nabla_{\square} D \Psi-\Lambda \tau
$$

where $\nabla U=\nabla_{\square} V=\nabla_{\square} W=\mathbf{0},\langle\Psi\rangle_{\square}=\langle\tau\rangle_{\square}=0$ and $\Psi$ and $\tau$ are d.p. The important derived scalars are $S_{\mathscr{S}} \boldsymbol{u}=0, S_{\mathscr{P}} \boldsymbol{u}=\nabla_{\square}^{2} \nabla^{2} \boldsymbol{\Psi}$ and $S_{\mathscr{g}} \boldsymbol{u}=-\nabla_{\square}^{2} \tau$, and $S_{\mathscr{P}_{\ell}} u=\kappa_{\ell}^{2}\left(\kappa_{\ell}^{2}-\mathrm{D}^{2}\right) \widetilde{\Psi}_{\ell}$ and $S_{\mathscr{T}_{\ell}} u=\kappa_{\ell}^{2} \widetilde{\tau}_{\ell}$.

Although the pressure gradient $\nabla P$ is d.p., the pressure needn't be. The most general d.p. form is $\nabla P=p_{x} \mathbf{i}_{x}+p_{y} \mathbf{i}_{y}+p_{z} \mathbf{i}_{z}+\nabla \varpi$, where $\nabla_{\square} p_{x}=\nabla p_{y}=$ $\nabla p_{z}=\mathbf{0},\langle\varpi\rangle_{\square}=0$, and $\varpi$ is d.p. The derived scalars are $S_{\mathscr{S}} \nabla P=\nabla^{2} \varpi$, $S_{\mathscr{S}_{\ell}} \nabla P=\left(\mathrm{D}^{2}-\kappa_{\ell}^{2}\right) \widetilde{\varpi}_{\ell}$, and $S_{\mathscr{G}} \nabla P=\mathrm{S}_{\mathscr{T}} \nabla P=0$.

3.2. Decomposition of the Stokes momentum equation In terms of the representations of Section 3.1, the decomposition (2.11)-(2.15) of the Stokes momentum equation (3.1) leads to four velocity equations

$$
\begin{aligned}
\mathrm{D}^{2} V & =p_{y}-\left\langle b_{y}\right\rangle_{\square}, \quad \mathrm{D}^{2} W=p_{z}-\left\langle b_{z}\right\rangle_{\square}, \\
-\nabla_{\square}^{2} \nabla^{4} \Psi & =-\nabla_{\square}^{2} b_{x}+\mathrm{D} \nabla_{\square} \cdot \boldsymbol{b} \equiv \mathrm{S}_{\mathscr{P}} \boldsymbol{b} \quad \text { and } \\
\nabla_{\square}^{2} \nabla^{2} \tau & =\boldsymbol{\Lambda} \cdot \boldsymbol{b} \equiv \mathrm{S}_{\mathscr{g}} \boldsymbol{b},
\end{aligned}
$$


three pressure equations

$$
\begin{aligned}
p_{x}-\left\langle p_{x}\right\rangle & =\left\langle b_{x}\right\rangle_{\square}-\left\langle b_{x}\right\rangle, \quad\left\langle p_{x}\right\rangle=\left\langle b_{x}\right\rangle \quad \text { and } \\
\nabla^{2} \varpi & =\nabla \cdot b-\langle\nabla \cdot b\rangle_{\square} \equiv S_{y} b,
\end{aligned}
$$

and the end condition

$$
\nabla_{\square}^{2} w=\nabla_{\square}^{2} \mathrm{D} \nabla^{2} \Psi+\nabla_{\square} \cdot \boldsymbol{b} \quad\left(x=x_{0}, x_{1}\right) .
$$

The end condition (3.6) provides a boundary condition for (3.5) whenever $\Psi$ is determined by (3.3) and velocity boundary conditions.

The poloidal (3.3) and toroidal (3.4) Stokes equations are related to the normal velocity-normal vorticity equations used by Chandrasekhar [4, pages 20-21] and others [2]; in the present notation, the nonmean part of the normal velocity is $-\nabla_{\square}^{2} \Psi$ and that of the normal vorticity is $-\nabla_{\square}^{2} \tau$.

3.2.1. Fourier decomposition of the Stokes equations Either by using the modification of Theorem 2.1 described in Section 2.3 or by taking the Fourier coefficients of the equations in Section 3.2, we can replace the poloidal (3.3), toroidal (3.4) and scaloidal (3.5) equations with (for all $\ell \neq 0$ )

$$
\begin{aligned}
\kappa_{\ell}^{2}\left(\kappa_{\ell}^{2}-\mathrm{D}^{2}\right)^{2} \tilde{\Psi}_{\ell} & =\mathrm{S}_{\mathscr{P}_{\ell}} b \equiv \kappa_{\ell}^{2}\left(e_{\ell}, b_{x}\right\rangle_{\square}+\mathrm{i} \kappa_{\ell} \mathrm{D}\left\langle e_{\ell}, b_{\eta_{\ell}}\right\rangle_{\square} \\
\kappa_{\ell}^{2}\left(\kappa_{\ell}^{2}-\mathrm{D}^{2}\right) \tilde{\tau}_{\ell} & =\mathrm{S}_{\mathscr{T}_{\ell}} b \equiv \mathrm{i} \kappa_{\ell}\left(e_{\ell}, b_{\zeta_{\ell}}\right\rangle_{\square}
\end{aligned}
$$

and

$$
\left(\mathrm{D}^{2}-\kappa_{\ell}^{2}\right) \widetilde{\varpi}_{\ell}=\mathrm{D}\left\langle e_{\ell}, b_{x}\right\rangle_{\square}+\mathrm{i} \kappa_{\ell}\left\langle e_{\ell}, b_{\eta_{\ell}}\right\rangle_{\square} \equiv \mathrm{S}_{\mathscr{S}_{\ell}} b .
$$

The end condition (3.6) becomes

$$
\kappa_{\ell}^{2} \widetilde{\varpi}_{\ell}=\kappa_{\ell}^{2} \mathrm{D}\left(\mathrm{D}^{2}-\kappa_{\ell}^{2}\right) \widetilde{\Psi}_{\ell}-\mathrm{i} \kappa_{\ell}\left\langle e_{\ell}, b_{\eta_{\ell}}\right\rangle_{\square} \quad\left(x=x_{0}, x_{1}\right) .
$$

3.3. Solution of the Stokes problem in a slot For a slot between solid walls at $x= \pm 1$, the boundary conditions are

$$
U=V=W=\tilde{\psi}_{\ell}=\mathrm{D} \tilde{\Psi}_{\ell}=\tilde{\tau}_{\ell}=0 .
$$

The mean velocity component $V$ is

$$
V(\xi)=\left\langle G(x ; \xi),\left\langle b_{y}\right\rangle_{\square}-p_{y}\right\rangle,
$$

where $G(x ; \xi)=\{1+\min (x, \xi)\}\{1-\max (x, \xi)\}$. The same solution applies to $W$ with the $y$ subscript replaced by $z$.

The Fourier toroidal component is $\tilde{\tau}_{\ell}(\xi)=\left\langle G(x ; \xi), \mathrm{S}_{\mathscr{\mathscr { T }}} \boldsymbol{b}\right\rangle$, where

$$
G(x ; \xi)=\frac{2 \sinh \kappa_{\ell}\{1+\min (x, \xi)\} \sinh \kappa_{\ell}\{1-\max (x, \xi)\}}{\kappa_{\ell}^{3} \sinh 2 \kappa_{\ell}} .
$$


3.3.1. Solution of the poloidal part of the Stokes problem The poloidal part is more complicated, being governed by (3.7), a fourth-, rather than second-, order equation; however, a solution can be obtained systematically by variation of parameters (see, for example, [6]).

A basis for the solution space of (3.7) with $S_{\mathscr{P}_{\ell}} b=0$ is

$$
\begin{aligned}
& p_{1}(x)=(1+x) \sinh \kappa_{\ell}(1+x), \\
& p_{2}(x)=\sinh 2 \kappa_{\ell} \sinh \kappa_{\ell}(1+x)-\kappa_{\ell}(1+x) \sinh \kappa_{\ell}(1-x), \\
& p_{3}(x)=(1-x) \sinh \kappa_{\ell}(1-x) \text { and } \\
& p_{4}(x)=\sinh 2 \kappa_{\ell} \sinh \kappa_{\ell}(1-x)-\kappa_{\ell}(1-x) \sinh \kappa_{\ell}(1+x) .
\end{aligned}
$$

Notice that $p_{1}, p_{2}, \mathrm{D} p_{1}$ and $\mathrm{D} p_{2}$ vanish at $x=-1$ while $p_{3}$ and $p_{4}$ satisfy the boundary conditions (3.9) at $x=1$. Then

$$
\tilde{\Psi}_{\ell}(\xi)=\left\langle G(x ; \xi), S_{\mathscr{P}_{\ell}} b\right\rangle
$$

where

$$
G(x ; \xi)= \begin{cases}+q_{3}(x) p_{3}(\xi)+q_{4}(x) p_{4}(\xi) & x<\xi \\ -q_{1}(x) p_{1}(\xi)-q_{2}(x) p_{2}(\xi) & x>\xi\end{cases}
$$

is the solution provided that

$$
\left[\begin{array}{cccc}
p_{1} & p_{2} & p_{3} & p_{4} \\
\mathrm{D} p_{1} & \mathrm{D} p_{2} & \mathrm{D}_{3} & \mathrm{D} p_{4} \\
\mathrm{D}^{2} p_{1} & \mathrm{D}^{2} p_{2} & \mathrm{D}^{2} p_{3} & \mathrm{D}^{2} p_{4} \\
\mathrm{D}^{3} p_{1} & \mathrm{D}^{3} p_{2} & \mathrm{D}^{3} p_{3} & \mathrm{D}^{3} p_{4}
\end{array}\right]\left[\begin{array}{c}
q_{1} \\
q_{2} \\
q_{3} \\
q_{4}
\end{array}\right]=\left[\begin{array}{c}
0 \\
0 \\
0 \\
1 / 2 \kappa_{\ell}^{2}
\end{array}\right] .
$$

Cramer's rule (see, for example, [1, page 55]) leads to

$$
\begin{aligned}
& q_{1}(x)=-W\left(p_{2}, p_{3}, p_{4} ; x\right) / 2 \kappa_{\ell}^{2} W\left(p_{1}, p_{2}, p_{3}, p_{4} ; x\right), \\
& q_{2}(x)=+W\left(p_{1}, p_{3}, p_{4} ; x\right) / 2 \kappa_{\ell}^{2} W\left(p_{1}, p_{2}, p_{3}, p_{4} ; x\right), \\
& q_{3}(x)=-W\left(p_{1}, p_{2}, p_{4} ; x\right) / 2 \kappa_{\ell}^{2} W\left(p_{1}, p_{2}, p_{3}, p_{4} ; x\right) \text { and } \\
& q_{4}(x)=+W\left(p_{1}, p_{2}, p_{3} ; x\right) / 2 \kappa_{\ell}^{2} W\left(p_{1}, p_{2}, p_{3}, p_{4} ; x\right),
\end{aligned}
$$

where $W$ stands for the Wronskian (see, for example, [1, page 132])

$$
W\left(p_{1}, p_{2}, p_{3} ; x\right) \equiv\left|\begin{array}{ccc}
p_{1} & p_{2} & p_{3} \\
\mathrm{D} p_{1} & \mathrm{D} p_{2} & \mathrm{D} p_{3} \\
\mathrm{D}^{2} p_{1} & \mathrm{D}^{2} p_{2} & \mathrm{D}^{2} p_{3}
\end{array}\right| .
$$

Since (3.7) is self-adjoint with constant coefficients, $W\left(p_{1}, p_{2}, p_{3}, p_{4} ; x\right)$ is a constant; a consequence of Abel's formula [18, page 203]. Here

$$
W\left(p_{1}, p_{2}, p_{3}, p_{4}\right)=-\kappa_{\ell}^{4}\left(\cosh 4 \kappa_{\ell}-1\right)\left(\cosh 4 \kappa_{\ell}-1-8 \kappa_{\ell}^{2}\right)
$$


and

$$
\begin{aligned}
& W\left(p_{2}, p_{3}, p_{4} ; x\right)=-\kappa_{\ell}^{3} \sinh 4 \kappa_{l} \sinh \kappa_{\ell}(1+x) \\
& +\kappa_{\ell}^{3} x\left(\cosh 4 \kappa_{l}-1\right) \cosh \kappa_{\ell}(1+x) \\
& +\left\{\left(\cosh 4 \kappa_{\ell}-1\right) \cosh 2 \kappa_{\ell}+2 \kappa_{\ell} \sinh 2 \kappa_{\ell}\right\} \kappa_{\ell}^{2} \sinh \kappa_{\ell}(1-x) \\
& -4 \kappa_{l}^{4}(1-x) \sinh 2 \kappa_{l} \cosh \kappa_{\ell}(1-x) \text {, } \\
& W\left(p_{1}, p_{3}, p_{4} ; x\right)=-2 \kappa_{\ell}^{2} \sinh 2 \kappa_{\ell} \sinh \kappa_{\ell}(1+x) \\
& +\kappa_{l}^{2} x\left(\cosh 4 \kappa_{l}-1\right) \cosh \kappa_{l}(1-x) \\
& +\left(\cosh 4 \kappa_{l}-1+\kappa_{l} \sinh 4 \kappa_{l}\right) \kappa_{\ell} \sinh \kappa_{\ell}(1-x) \\
& -4 \kappa_{l}^{3}(1-x) \sinh 2 \kappa_{l} \cosh \kappa_{l}(1+x) \text {, } \\
& W\left(p_{1}, p_{2}, p_{4} ; x\right)=\kappa_{l}^{3} \sinh 4 \kappa_{l} \sinh \kappa_{l}(1-x) \\
& +\kappa_{\ell}^{3} x\left(\cosh 4 \kappa_{\ell}-1\right) \cosh \kappa_{\ell}(1-x) \\
& -\left\{\left(\cosh 4 \kappa_{l}-1\right) \cosh 2 \kappa_{l}+2 \kappa_{l} \sinh 2 \kappa_{l}\right\} \kappa_{l}^{2} \sinh \kappa_{l}(1+x) \\
& +4 \kappa_{\ell}^{4}(1+x) \sinh 2 \kappa_{l} \cosh \kappa_{\ell}(1+x) \text { and } \\
& W\left(p_{1}, p_{2}, p_{3} ; x\right)=2 \kappa_{\ell}^{2} \sinh 2 \kappa_{l} \sinh \kappa_{l}(1-x) \\
& +\kappa_{\ell}^{2} x\left(\cosh 4 \kappa_{\ell}-1\right) \cosh \kappa_{\ell}(1+x) \\
& -\left\{\cosh 4 \kappa_{\ell}-1+\kappa_{\ell} \sinh 4 \kappa_{\ell}\right\} \kappa_{\ell} \sinh \kappa_{\ell}(1+x) \\
& +4 \kappa_{\ell}^{3}(1+x) \sinh 2 \kappa_{\ell} \cosh \kappa_{\ell}(1-x) \text {. }
\end{aligned}
$$

\subsection{Examples of Stokes solutions}

3.4.1. Base flow components If the body force is analytic in $x$ so that

$$
f(x) \equiv\left\langle b_{y}\right\rangle_{\square}-p_{y}=\sum_{k=0}^{\infty} f_{k} x^{k}
$$

then (3.10) gives

$$
V(x)=\sum_{k=0}^{\infty} f_{k} \frac{\left\{1+(-1)^{k}\right\} / 2+\left[1-(-1)^{k}\right\} x / 2-x^{k+2}}{(k+1)(k+2)} .
$$

Important special cases include $f(x)=1: V(x)=\left(1-x^{2}\right) / 2$, that is, Poiseuille flow [7, page 582]; and $f(x)=x: V(x)=x\left(1-x^{2}\right) / 6$, which arises in natural convection in a vertical slot [20].

3.4.2. Fundamental poloidal velocity fields Consider the force distribution $b=\kappa_{\ell}^{-2} \delta(x-s) \cos \kappa_{\ell} \eta_{\ell} \mathbf{i}_{x}$, where $s$ is a constant $-1<s<1$. We have $S_{\mathscr{P}_{\ell}} b=$ $\mathrm{S}_{\mathscr{P}_{(-2)}} b=\delta(x-s) / 2$, so that

$$
2 \widetilde{\Psi}_{l}(x)= \begin{cases}-q_{1}\left(x_{0}\right) p_{1}(x)-q_{2}\left(x_{0}\right) p_{2}(x) & x<s \\ +q_{3}\left(x_{0}\right) p_{3}(x)+q_{4}\left(x_{0}\right) p_{4}(x) & x>s,\end{cases}
$$




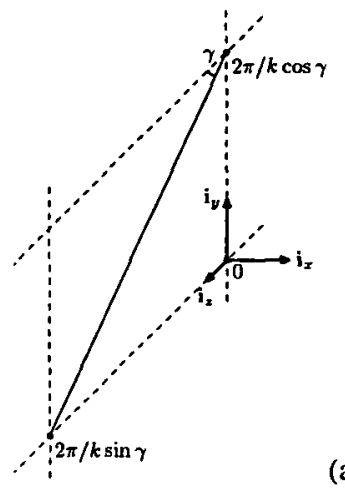

(a)
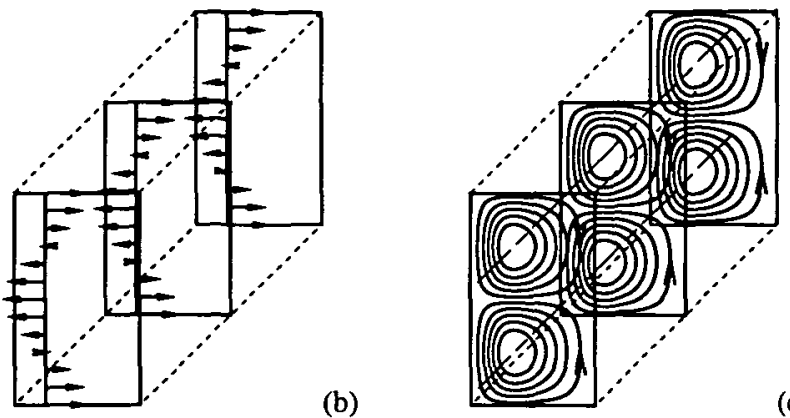

(c)

FIGURE 1. Poloidal rolls from the example in Section 3.4.2 with $k=2, \gamma=\pi / 8, l_{y}=1, l_{z}=0$ and $s=-1 / 2$ : (a) geometry; (b) schematic of the force distribution; (c) stream-lines (solid) and roll axis (chain-line). Also marked in (b) and (c) are two $y z$-period cells and $|x|=1$ (dashed) and the planes $\zeta_{\ell}=0, \pi \cos \beta_{\ell} / k \sin \gamma$ and $2 \pi \cos \beta_{\ell} / k \sin \gamma$ (solid).

$\widetilde{\Psi}_{-\ell}=\widetilde{\Psi}_{\ell}$ and the stream-function in any $x \eta_{\ell}$-plane is $\psi=-2 \kappa_{\ell} \widetilde{\Psi}_{\ell} \sin \kappa_{\ell} \eta_{\ell}$. This signifies a spatially periodic flow consisting of counterrotating rolls with axes parallel to the walls (Figure 1).

Note that the same poloidal velocity field would be generated by the force distribution $\kappa_{\ell}^{-2} H(x-s) \sin \kappa_{\ell} \eta_{\ell} i_{\eta_{\ell}}$, where $H$ is Heaviside's step function. Neither of these force distributions is purely poloidal; they have the same scalar $S_{\mathscr{P}}$ but different scaloidal parts and so give rise to different pressure distributions.

3.4.3. Fundamental toroidal velocity fields The force distribution

$$
\boldsymbol{b}=\kappa_{\ell}^{-1} \delta(x-s) \sin \kappa_{\ell} \eta_{\ell} \mathbf{i}_{\zeta \ell}
$$

gives rise to a toroidal velocity field with scalar $\tau=2 \tilde{\tau}_{\ell} \cos \kappa_{\ell} \eta_{\ell}$, where $\tilde{\tau}_{\ell}(x)=$ $G(x ; s)$ with $G$ as in (3.11). The velocity field, $u=2 \kappa_{\ell} \tilde{\tau}_{\ell} \sin \kappa_{\ell} \eta_{\ell} i_{\zeta \ell}$, is unidirectional as shown in Figure 2. This force distribution causes no other velocity field and makes no contribution to the pressure.

Note that by the properties and construction of the Green's functions, these poloidal and toroidal examples have continuous velocity fields but discontinuous velocity derivatives beyond a certain order, due to the delta-spike in the force distributions.

\section{Conclusions}

In general, the two main difficulties in solving the Navier-Stokes equations are the incompressibility constraint and the nonlinearity of the advection terms. The Stokes equations share the former and lack the latter. The quinquepartite decomposition 


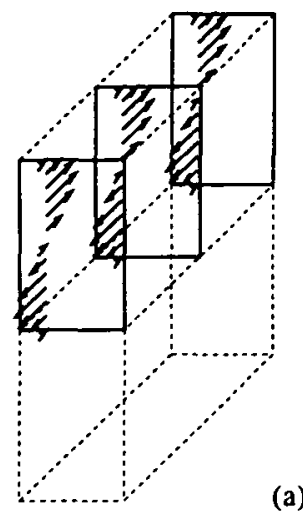

(a)

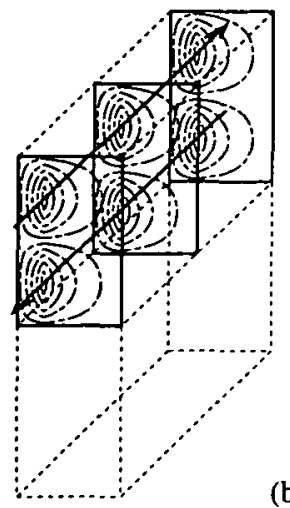

(b)

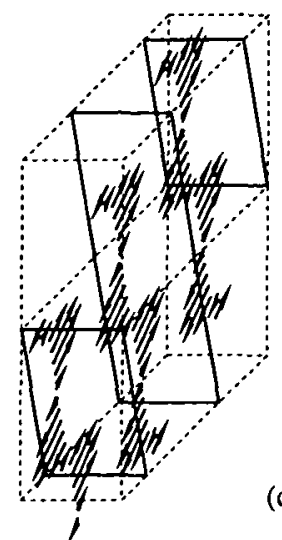

(c)

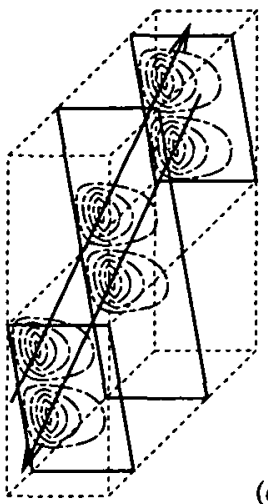

(d)

FIGURE 2. The example toroidal field of Section $3.4 .3, l_{y}=1$; projection and periodicity $(k$ and $\gamma$ ) as in Figure 1: (a, b) $l_{z}=0$; (c, d) $l_{z}=1$; (a, c) schematic of force distribution; (b, d) chain-lines mark vortex lines (which are also contours of constant velocity, by [9, Theorem 2]) and solid lines the stream-lines of maximum velocity.

proves to be a effective way of dealing with the constraint, as shown in the past for the poloidal-toroidal decomposition in spherical geometries $[7,11,13]$.

Although we have only treated the Stokes and generalised Stokes equations, the decomposition presented can be applied to any vector field equation with periodic boundary conditions in two directions on a domain bounded in the third orthogonal direction. It may be expected to be useful in other cases where a divergence-free vector field is involved and the divergence-free subspaces $\mathscr{B}, \mathscr{P}$ and $\mathscr{T}$ are invariant under the linear operators involved. Two such examples are

$$
\frac{\partial \boldsymbol{H}}{\partial t}=-\eta \nabla \times(\nabla \times H) \text { and } \nabla \cdot \boldsymbol{H}=0,
$$

which govern the decay of a magnetic field $\boldsymbol{H}$ in a motionless medium with uniform resistivity $\eta$ [4, pages 147-150] and $u+K \nabla P=b$ and $\nabla \cdot u=0$ which govern groundwater flow [16].

\section{Appendix A. Generalised Stokes problem}

The decomposition of the generalised Stokes problem (1.1)-(1.2) is

$$
\begin{aligned}
\left(\mathrm{D}^{2}-\alpha\right) V & =p_{y}-\left\langle b_{y}\right\rangle_{\square}, \quad\left(\mathrm{D}^{2}-\alpha\right) W=p_{z}-\left\langle b_{z}\right\rangle_{\square}, \\
\nabla_{\square}^{2} \nabla^{2}\left(\alpha-\nabla^{2}\right) \Psi & =-\nabla_{\square}^{2} b_{x}+\mathrm{D} \nabla_{\square} \cdot \boldsymbol{b} \equiv \mathrm{S}_{\mathscr{S}} b, \\
-\nabla_{\square}^{2}\left(\alpha-\nabla^{2}\right) \tau & =\boldsymbol{\Lambda} \cdot \boldsymbol{b} \equiv \mathrm{S}_{\mathscr{T}} b, \\
p_{x}-\left\langle p_{x}\right\rangle & =\left\langle b_{x}\right\rangle_{\square}-\left\langle b_{x}\right\rangle, \quad\left\langle p_{x}\right\rangle=\left\langle b_{x}\right\rangle-\alpha U \quad \text { and } \\
\nabla^{2} \varpi & =\nabla \cdot \boldsymbol{b}-\langle\nabla \cdot \boldsymbol{b}\rangle_{\square} \equiv \mathrm{S}_{\mathscr{S}} \boldsymbol{b}
\end{aligned}
$$


with the end condition $(x= \pm 1)$

$$
\nabla_{\square}^{2} \varpi=\nabla_{\square}^{2} \mathrm{D}\left(\nabla^{2}-\alpha\right) \Psi+\nabla_{\square} \cdot \boldsymbol{b} .
$$

For the Fourier coefficients $(\ell \neq 0)$, the poloidal (A.1) and toroidal (A.2) equations can be replaced with

$$
\kappa_{\ell}^{2}\left(\kappa_{\ell}^{2}-\mathrm{D}^{2}\right)\left(\lambda_{\ell}^{2}-\mathrm{D}^{2}\right) \widetilde{\Psi}_{\ell}=\mathrm{S}_{\mathscr{P}_{\ell}} b \quad \text { and } \quad \kappa_{\ell}^{2}\left(\lambda_{\ell}^{2}-\mathrm{D}^{2}\right) \tilde{\tau}_{\ell}=\mathrm{S}_{\mathscr{T}_{l}} b
$$

and the end condition (A.3) with $\kappa_{\ell}^{2} \widetilde{\varpi}_{\ell}=\kappa_{\ell}^{2} \mathrm{D}\left(\mathrm{D}^{2}-\lambda_{\ell}^{2}\right) \widetilde{\Psi}_{\ell}-\mathrm{i} \kappa_{\ell}\left(e_{\ell}, b_{\eta_{\ell}}\right)_{0}$, where $\lambda_{\ell} \equiv \sqrt{\alpha+\kappa_{\ell}^{2}}$. The Fourier scaloidal equation (3.8) is unchanged by the addition of the generalised $\alpha$ term.

The solution for $\boldsymbol{u}=\mathbf{0}$ on $\boldsymbol{x}= \pm 1$ has mean velocity components

$$
V(\xi)=\left\langle G(x ; \xi),\left\langle b_{y}\right\rangle_{\square}-p_{y}\right\rangle \text { and } W(\xi)=\left\langle G(x ; \xi),\left\langle b_{z}\right\rangle_{\square}-p_{z}\right\rangle
$$

where

$$
G(x ; \xi)=\frac{2 \sinh \sqrt{\alpha}\{1+\min (x, \xi)\} \sinh \sqrt{\alpha}\{1-\max (x, \xi)\}}{\sqrt{\alpha} \sinh 2 \sqrt{\alpha}},
$$

and the toroidal scalar has Fourier coefficients $\tilde{\tau}_{\ell}(\xi)=\left\langle G(x ; \xi), S_{\mathscr{T}_{\ell}} b\right\rangle$, where

$$
G(x ; \xi)=\frac{2 \sinh \lambda_{\ell}\{1+\min (x, \xi)\} \sinh \lambda_{\ell}\{1-\max (x, \xi)\}}{\kappa_{\ell}^{2} \lambda_{\ell} \sinh 2 \lambda_{\ell}} .
$$

The $\widetilde{\Psi}_{\ell}$ are given by (3.12)-(3.13) and (3.14)-(3.17) with

$$
\begin{aligned}
& p_{1}(x)=\lambda_{l} \sinh \kappa_{l}(1+x)-\kappa_{\ell} \sinh \lambda_{l}(1+x), \\
& p_{2}(x)=\cosh \kappa_{\ell}(1+x)-\cosh \lambda_{l}(1+x), \\
& p_{3}(x)=\lambda_{\ell} \sinh \kappa_{l}(1-x)-\kappa_{\ell} \sinh \lambda_{l}(1-x) \quad \text { and } \\
& p_{4}(x)=\cosh \kappa_{\ell}(1-x)-\cosh \lambda_{l}(1-x) .
\end{aligned}
$$

\section{References}

[1] A. C. Aitken, Determinants and Matrices, 9th ed. (Oliver and Boyd, Edinburgh, 1956).

[2] D. J. Benney and L. H. Gustavsson, "A new mechanism for linear and nonlinear hydrodynamic stability", Stud. Appl. Math. 64 (1981) 185-209.

[3] J. Cahouet and J.-P. Chabard, "Some fast 3D finite element solvers for the generalized Stokes problem", Intl J. Numer. Methods Fluids 8 (1988) 869-895.

[4] S. Chandrasekhar, Hydrodynamic and Hydromagnetic Stability (Dover, New York, 1981).

[5] G. P. Galdi and C. G. Simader, "Existence, uniqueness and $l^{q}$-estimates for the Stokes problem in an exterior domain", Arch. Rational Mech. Anal. 112 (4) (1990) 291-318. 
[6] W. Kaplan, Elements of Ordinary Differential Equations (Addison-Wesley, Reading, Massachusetts, 1964).

[7] H. Lamb, Hydrodynamics, 6th ed. (Cambridge University Press, Cambridge, 1932).

[8] G. D. McBain, "Plane poloidal-toroidal decomposition of doubly periodic vector fields. Part 1. Fields with divergence", ANZIAM J. 47 (2005) 21-38.

[9] G. D. McBain, "Fully developed laminar buoyant flow in vertical cavities and ducts of bounded section", J. Fluid Mech. 401 (1999) 365-377.

[10] G. D. McBain, "Vapour transport across gas-filled enclosures", Ph. D. Thesis, James Cook University, Townsville, North Queensland, 1999.

[11] G. D. McBain, "Convection in a horizontally heated sphere", J. Fluid Mech. 438 (2001) 1-10.

[12] B. R. Munson and D. D. Joseph, "Viscous incompressible flow between concentric rotating spheres. Part I. Basic flow.", J. Fluid Mech. 49 (1971) 289-303.

[13] D. Palaniappan, S. D. Nigam and T. Amaranth, "A theorem for a fluid sphere in Stokes flow", J. Austral. Math. Soc. Ser. B 35 (1994) 335-347.

[14] C. Sabbah, M. Y. Forestier and R. Pasquetti, "Filtering of the pressure spurious modes in $\mathbb{P}_{n}-\mathbb{P}_{n}$ spectral approximations of the Stokes system", Comput. \& Fluids 31 (2002) 815-823.

[15] C. Sabbah and R. Pasquetti, "A divergence-free multi-domain spectral solver of the Navier-Stokes equations in geometries of high aspect ratio", J. Comput. Phys. 139 (1998) 359-379.

[16] R. Scheichl, "Decoupling three-dimensional mixed problems using divergence-free finite elements", SIAM J. Sci. Comput. 23 (2002) 1752-1776.

[17] B. J. Schmitt and W. von Wahl, "Decomposition of solenoidal fields into poloidal fields, toroidal fields and the mean flow. Applications to the Boussinesq equations", Lecture Notes in Math. 1530 (1992) 291-305.

[18] I. Stakgold, Green's Functions and Boundary Value Problems, 2nd ed. (Wiley-Interscience, New York, 1998).

[19] N. Stokes, N. Barton and L. Gross, "Inexact preconditioned Uzawa methods as a general approach to calculations in computational fluid dynamics", Paper presented to the 11th Computational Techniques and Applications Conference, Sydney, New South Wales, 2003.

[20] L. Waldmann, "Zur Theorie des Gastrennungsverfahrens von Clusius und Dickel", Die Naturwissenschaften 27 (14) (1939) 230-231. 